\title{
NATURAL MATES OF NON-NULL FRENET CURVES IN MINKOWSKI 3-SPACE
}

\author{
ALEV KELLECI \\ Department of Mathematics, Firat University, Turkey \\ Corresponding author: alevkelleci@hotmail.com
}

Received May 17, 2020

\begin{abstract}
Aвstract. In this paper, we define the natural mate of a non-null Frenet curve in Minkowski 3-spaces. The main aim of that paper is to find some relationships between a non-null Frenet curve and its natural mate. In this context, we obtain some results for the natural mate of a non-null Frenet curve to be a slant helix, a spherical curve, or a curve of constant curvature. Finally, as an application, we give an explicit example for natural mates.

2010 Mathematics Subject Classification. 53B25, 53A35, 53C50.

Key words and phrases. non-null curve; rectifying curves; natural mate; spherical curves; slant helix; Minkowski space.
\end{abstract}

\section{InTRODUCTION}

In the study of the fundamental theory and the characterizations of space curves, the corresponding relations between the curves are the very interesting and important problem not only in Euclidean space but also Lorentzian space. One of the most popular among these curves is the Frenet curve. If a curve $\gamma$ is a Frenet curve, then its curvature $\kappa>0$ and torsion $\tau \neq 0$, studied in [7-11] by many geometers. Some important kinds of these curves are slant helices, spherical curves, rectifying curves, characterized in $[1,3,4,12,13,15,18-21]$. Since there exist three kinds of curves (time-like, space-like, and null or light-like curves) depending on their causal characters in Lorentzian space, working in Lorentzian space is more complicated than working in Euclidean space. Also, it is well-known that the studies of space-like curves and time-like curves have many analogies and similarities because they have the natural

DOI: $10.28924 / A P J M / 7-18$

(2020 Asia Pacific Journal of Mathematics 
geometric invariant parameter by the arc-length parameter which normalizes the tangent vector, [17].

Recently, the theory of the associated curve of a given curve has been one of the interesting topics. Many geometers have investigated this problem from different viewpoints: For example, the Bertrand partner and the Mannheim partner curves in Minkowski 3-space, are two important types of associated curves, characterized by the curvature and the torsion, (see in $[2,5,6,16])$. In [8], the authors studied also the general helices and the slant helices in Minkowski 3-spaces, by using some special associated curves of a given curve. Subsequently, they studied the Euclidean version in [7]. They called the special associated curve the principal-directional (-donor) curve and the binormal-directional (-donor) curve. These notions gave us a certain method of constructing the general helices and the slant helices (see $[7,8])$. In [10], Deshmukh, Chen and Alghanemi studied a new type associated curve called as the natural mate of a Frenet curve in Euclidean 3-space, closely related with the principal-directional (-donor) curve defined in $[7,8]$. They characterized these curves and also gave new results for them.

In that paper, firstly we define the concept of a natural mate for non-null Frenet curves in Minkowski 3-space by moving from the notion of natural mate defined in [10]. In Sect. 4, we give the Lorentzian character and type of the natural mate curve and show that the natural mate of the non-null Frenet curves in Minkowski 3-space is unique in Theorem 4.1. Also, in the last section we obtain some relationship between these natural mates and as an application, we give an explicit example for them with their graph.

All geometric objects under consideration are smooth and curves are regular unless otherwise stated.

\section{Preliminaries}

In this section, we would like to give a brief summary of basic definitions, facts and equations in the theory of curves and surfaces in Minkowski 3-space (see for detail, $[14,17]$ ).

Let $\mathbb{E}_{1}^{3}$ denote the Minkowski 3-space with the canonical Lorentzian metric tensor given by

$$
\langle\cdot, \cdot\rangle=-d x_{1}^{2}+d x_{2}^{2}+d x_{3}^{2}
$$

where $\left(x_{1}, x_{2}, x_{3}\right)$ are rectangular coordinates of the points of $\mathbb{E}_{1}^{3}$.

The causality of a vector in a Minkowski space is defined as follows. A non-zero vector $v$ in $\mathbb{E}_{1}^{3}$ is said to be space-like, time-like and light-like (null) regarding to $\langle v, v\rangle>0,\langle v, v\rangle<0$ and 
$\langle v, v\rangle=0$, respectively. We consider the zero vector as a space-like vector. Note that $v$ is said to be causal if it is not space-like. Two non-zero vectors $u$ and $v$ in $\mathbb{E}_{1}^{3}$ are said to be orthogonal if $\langle u, v\rangle=0$.

A set of $\left\{e_{1}, e_{2}, e_{3}\right\}$ of vectors in $\mathbb{E}_{1}^{3}$ is called an orthonormal frame if it satisfies that

$$
\left\langle e_{1}, e_{1}\right\rangle=-1, \quad\left\langle e_{2}, e_{2}\right\rangle=\left\langle e_{3}, e_{3}\right\rangle=1, \quad\left\langle e_{i}, e_{j}\right\rangle=0, \quad i \neq j .
$$

For two non-zero vectors $u=\left(u_{1}, u_{2}, u_{3}\right)$ and $v=\left(v_{1}, v_{2}, v_{3}\right)$ in $\mathbb{E}_{1}^{3}$, we define the (Lorentzian) vector product of $u$ and $v$ as following:

$$
u \times v=\left(u_{3} v_{2}-u_{2} v_{3}, u_{3} v_{1}-u_{1} v_{3}, u_{1} v_{2}-u_{2} v_{1}\right) .
$$

One check that the vector product is skew-symmetric, i.e., $u \times v=-v \times u$.

A curve $\gamma=\gamma(t)$ in $\mathbb{E}_{1}^{3}$ is said to be space-like, time-like or null (light-like) if its tangent vector field $\gamma^{\prime}(t)$ is space-like, time-like or null (light-like), respectively, for all $t$.

Let $\gamma$ be a non-null curve in $\mathbb{E}_{1}^{3}$ parametrized by arc-length, i.e., $\left|\left\langle\gamma^{\prime}, \gamma^{\prime}\right\rangle\right|=1$, and we suppose that $\left|\left\langle\gamma^{\prime \prime}, \gamma^{\prime \prime}\right\rangle\right| \neq 0$. Then this curve $\gamma$ induces a Frenet frame $\left\{T=\gamma^{\prime}, N=\frac{\gamma^{\prime \prime}}{\sqrt{\left|\left\langle\gamma^{\prime \prime}, \gamma^{\prime \prime}\right\rangle\right|}}, B=T \times N\right\}$ satisfying the following Frenet equations:

$$
\left[\begin{array}{c}
T^{\prime} \\
N^{\prime} \\
B^{\prime}
\end{array}\right]=\left[\begin{array}{ccc}
0 & \kappa \varepsilon_{1} & 0 \\
-\kappa \varepsilon_{0} & 0 & -\tau \varepsilon_{0} \varepsilon_{1} \\
0 & -\tau \varepsilon_{1} & 0
\end{array}\right]\left[\begin{array}{l}
T \\
N \\
B
\end{array}\right]
$$

where $\langle T, T\rangle=\varepsilon_{0},\langle N, N\rangle=\varepsilon_{1},\langle B, B\rangle=-\varepsilon_{0} \varepsilon_{1},\left\langle T^{\prime}, N\right\rangle=\kappa$ and $\left\langle N^{\prime}, B\right\rangle=\tau$. The vector fields $T, N, B$ and the functions $\kappa, \tau$ are called the tangent, principal normal, binormal and curvature and torsion of $\gamma$, respectively. Accordingly, the Frenet frame of $\gamma$ satisfies

$$
T \times N=B, \quad N \times B=-\varepsilon_{1} T, \quad B \times T=-\varepsilon_{0} N .
$$

In (2.1), if $\varepsilon_{0}=1$ or $\varepsilon_{0}=-1$, then $\gamma$ is space-like or time-like, respectively. A space-like curve $\gamma$ satisfying (2.1) is said to be type 1 or type 2 if $\varepsilon_{1}=1$ or $\varepsilon_{1}=-1$, respectively.

When the Frenet frame moves along a curve in $\mathbb{E}_{1}^{3}$, there exist an axis of instantaneaus frame's rotation (Darboux). The direction of such axis is given by Darboux vector. If $\gamma$ is a unit speed non-null curve with the Frenet-Serret apparatus $\left\{\kappa_{\gamma}, \tau_{\gamma}, T_{\gamma}, N_{\gamma}, B_{\gamma}\right\}$, the Darboux vector of $\gamma$ is that

$$
D_{\gamma}(s)=-\varepsilon_{0} \varepsilon_{1} \tau_{\gamma}(s) T_{\gamma}(s)-\varepsilon_{0} \varepsilon_{1} \kappa_{\gamma}(s) B_{\gamma}(s)
$$


whose length is given by

$$
\omega_{\gamma}=\sqrt{\left|\varepsilon_{0} \tau_{\gamma}^{2}-\varepsilon_{0} \varepsilon_{1} \kappa_{\gamma}^{2}\right|} .
$$

Note that the Darboux vector defined in (2.2) is also called as the centrode of $\gamma$ in $\mathbb{E}_{1}^{3}$. Thus, the Darboux equations are defined as

$$
\begin{gathered}
T_{\gamma}^{\prime}=D_{\gamma} \times T_{\gamma}, \\
N_{\gamma}^{\prime}=D_{\gamma} \times N_{\gamma}, \\
B_{\gamma}^{\prime}=D_{\gamma} \times B_{\gamma} .
\end{gathered}
$$

The co-centrode of $\gamma$ from the point of [10] is given by

$$
D_{\gamma}^{*}(s)=-\varepsilon_{0} \varepsilon_{1} \tau_{\gamma}(s) B_{\gamma}(s)-\varepsilon_{0} \kappa_{\gamma}(s) T_{\gamma}(s),
$$

which is exactly the derivative of the principal normal vector of $\gamma$ defined in (2.1).

Recently, in [10] authors have introduced the concept of the natural mate of a curve in $\mathbb{E}^{3}$. Motivated by what happens in Euclidean ambient space, we would like to give next definition:

Definition 2.1. Let $\gamma: I \rightarrow \mathbb{E}_{1}^{3}$ be a non-null Frenet curve in $\mathbb{E}_{1}^{3}$ parametrized by the arc-length parameter with Frenet frame $\left\{T_{\gamma}, N_{\gamma}, B_{\gamma}\right\}$. A curve $\beta: I \rightarrow \mathbb{E}_{1}^{3}$ is called the natural mate of the curve $\gamma$, if the tangent $T_{\beta}$ is equal to $N_{\gamma}$, i.e., $T_{\beta}=N_{\gamma}$.

\section{SOME KNOWN RESULTS}

In this section we would like to give some theorems obtained before, which will play an important role in the proofs of our results.

Definition 3.1. [13] A unit speed non-null curve is called rectifying curve if its position vector $x: I \rightarrow \mathbb{E}_{1}^{3}$ always lie in its rectifying plane, i.e., it satisfies

$$
x(s)=\lambda(s) T(s)+\mu(s) B(s)
$$

where some functions $\lambda$ and $\mu$.

Theorem 3.1. [13] Let $\gamma=\gamma(s)$ be a unit speed non-null curve in $\mathbb{E}_{1}^{3}$, with a space-like or a time-like rectifying plane and with the curvature $\kappa(s)>0$. Then up to the isometries of $\mathbb{E}_{1}^{3}$, the curve $\gamma$ is rectifying if and only if there holds

$$
\tau(s) / \kappa(s)=c_{1} s+c_{2}
$$

where $c_{1} \in R_{0}, c_{2} \in R$. 
Definition 3.2. [1] A unit speed non-null curve is called slant helix curve if there exists a non-zero constant vector field $U$ in $\mathbb{E}_{1}^{3}$ such that $\langle N(s), U\rangle$ is constant.

Theorem 3.2. [1] Let $\gamma$ be a unit speed non-null curve in $\mathbb{E}_{1}^{3}$. Then $\gamma$ is a slant helix if and only if the function

$$
\sigma_{\gamma}=\frac{\kappa_{\gamma}^{2}}{\left(\omega_{\gamma}\right)^{3}}\left(\frac{\tau}{\kappa}\right)^{\prime}
$$

is constant. Here $\omega_{\gamma}=\sqrt{\left|\varepsilon_{0} \tau_{\gamma}^{2}-\varepsilon_{0} \varepsilon_{1} \kappa_{\gamma}^{2}\right|}$ defined in (2.3).

Theorem 3.3. [18-20] Let $\gamma$ be a unit speed non-null curve in $\mathbb{E}_{1}^{3}$ with a curvature $\kappa(s) \neq 0$ and a torsion $\tau(s) \neq 0$ for each $s \in I \subset \mathbb{R}$. Then, $\gamma$ lies on the Lorentzian sphere of radius $r \in \mathbb{R}^{+}$if and only if

$$
\varepsilon_{1}{\frac{1}{\kappa_{\gamma}}}^{2}-\varepsilon_{0} \varepsilon_{1}\left[\frac{1}{\tau_{\gamma}}\left(\frac{1}{\kappa_{\gamma}}\right)^{\prime}\right]^{2}=r^{2}
$$

holds, where $\left\langle T_{\gamma}, t_{\gamma}\right\rangle=\varepsilon_{1}$ and $\left\langle N_{\gamma}, N_{\gamma}\right\rangle=\varepsilon_{1}$.

Theorem 3.4. [18-20] Let $\gamma$ be a unit speed non-null curve in $\mathbb{E}_{1}^{3}$. Then, $\gamma$ is a spherical curve, i.e. $\gamma$ lies on the Lorentzian sphere, if and only if

$$
\frac{\tau_{\gamma}}{\kappa_{\gamma}}=\varepsilon_{1}\left[\frac{1}{\tau_{\gamma}}\left(\frac{1}{\kappa_{\gamma}}\right)^{\prime}\right]^{\prime}
$$

holds, where $\tau_{\gamma}, \kappa_{\gamma}$ do not vanish and $\left\langle N_{\gamma}, N_{\gamma}\right\rangle=\varepsilon_{1}$.

\section{Natural Mates of NON-NUll Frenet CURVES}

In this section, first we recall the following results from [8].

Theorem 4.1. Let $\gamma$ be a non-null Frenet curve in $\mathbb{E}_{1}^{3}$ with Frenet-Serret apparatus $\left\{\kappa_{\gamma}, \tau_{\gamma}, T_{\gamma}, N_{\gamma}, B_{\gamma}\right\}$. Then, there exist a unit speed non-null curve $\beta$ with Frenet-Serret apparatus $\left\{\kappa_{\beta}, \tau_{\beta}, T_{\beta}, N_{\beta}, B_{\beta}\right\}$ given by

$$
\begin{array}{r}
\kappa_{\beta}=\varepsilon_{2} \omega_{\gamma}, \quad \tau_{\beta}=-\varepsilon_{0} \varepsilon_{1} \sigma_{\gamma} \omega_{\gamma}, \\
T_{\beta}=N_{\gamma}, \quad N_{\beta}=\frac{D_{\gamma}^{*}}{\omega_{\gamma}}, \quad B_{\beta}=\frac{D_{\gamma}}{\omega_{\gamma}}
\end{array}
$$

where $D_{\gamma}, \omega_{\gamma}, D_{\gamma}^{*}, \sigma_{\gamma}$ are defined in (2.2), (2.3), (2.5), (3.2), respectively.

Proof. Assume that $\gamma$ be a unit speed non-null Frenet curve in $\mathbb{E}_{1}^{3}$. Obviously, the set of $\left\{N_{\gamma}, \frac{D_{\gamma}^{*}}{\omega_{\gamma}}, \frac{D_{\gamma}}{\omega_{\gamma}}\right\}$ is an orthonormal frame along $\gamma$ in $\mathbb{E}_{1}^{3}$, where $D_{\gamma}, \omega_{\gamma}, D_{\gamma}^{*}$ are defined in (2.2), 
(2.3), (2.5), respectively. Assume that $\left\langle N_{\gamma}, N_{\gamma}\right\rangle=\varepsilon_{1}$ and $\left\langle\frac{D_{\gamma}^{*}}{\omega_{\gamma}}, \frac{D_{\gamma}^{*}}{\omega_{\gamma}}\right\rangle=\varepsilon_{2}$ and $\left\langle\frac{D_{\gamma}}{\omega_{\gamma}}, \frac{D_{\gamma}}{\omega_{\gamma}}\right\rangle=-\varepsilon_{1} \varepsilon_{2}$. By considering the function $\sigma_{\gamma}$ as defined in (3.2) and using, we get

$$
\left(\frac{\kappa_{\gamma}}{\omega_{\gamma}}\right)^{\prime}=\varepsilon_{0} \varepsilon_{1} \varepsilon_{2} \tau_{\gamma} \sigma_{\gamma} \quad \text { and } \quad\left(\frac{\tau_{\gamma}}{\omega_{\gamma}}\right)^{\prime}=\varepsilon_{0} \varepsilon_{1} \kappa_{\gamma} \sigma_{\gamma} .
$$

By using the last results and the Frenet formulas given in (2.1), we get

$$
N_{\gamma}{ }^{\prime}=\varepsilon_{2} D_{\gamma}^{*}, \quad\left(\frac{D_{\gamma}^{*}}{\omega_{\gamma}}\right)^{\prime}=-\varepsilon_{1} \varepsilon_{2} \omega_{\gamma} N_{\gamma}+\varepsilon_{0} \varepsilon_{2} \sigma_{\gamma} \omega_{\gamma}\left(\frac{D_{\gamma}}{\omega_{\gamma}}\right), \quad\left(\frac{D_{\gamma}}{\omega_{\gamma}}\right)^{\prime}=\varepsilon_{0} \varepsilon_{1} \varepsilon_{2} \sigma_{\gamma} \omega_{\gamma}\left(\frac{D_{\gamma}^{*}}{\omega_{\gamma}}\right) .
$$

By virtue of existence theorem, the equations obtained in (4.2) make assure that there exists a unit speed non-null curve $\beta$ in $\mathbb{E}_{1}^{3}$ with Frenet-Serret apparatus $\left\{\kappa_{\beta}, \tau_{\beta}, T_{\beta}, N_{\beta}, B_{\beta}\right\}$ described as in (4.1). Inversely, let $\beta$ is a unit speed non-null curve such that $\beta$ is tangent to $N_{\gamma}$ at each $s \in I$. Thus it follows from the Frenet frame of the non-null curve $\gamma$ that the Frenet-Serret apparatus of $\beta$ must be given in the way given above.

Remark 4.1. We would like to note that in [10], the authors explained why they chose to name the pair of $\{\gamma, \beta\}$ as the natural mates in $\mathbb{E}^{3}$. For the same reason, we will call the curve $\beta$ as the natural mate of $\gamma$ in $\mathbb{E}_{1}^{3}$ instead of the principal-direction curve defined in [8].

\section{Some Relationship between the Natural mates}

Firstly, we give the following consequences obtained easily from Theorem 4.1.

Corollary 5.1 ( [8], Lemma 4.1). A non-null Frenet curve $\gamma$ in $\mathbb{E}_{1}^{3}$ is a general helix with $\tau_{\gamma} \neq \kappa_{\gamma}$ if and only if its natural mate $\beta$ is a plane curve.

Corollary 5.2 ( [8], Corollary 4.7). A space-like Frenet curve $\gamma$ of type1 in $\mathbb{E}_{1}^{3}$ is a helix with $|\kappa|>|\tau|$ or $|\kappa|<|\tau|$ if and only if its space-like natural mate $\beta$ is a circle in $\mathbb{E}^{2}$ or in $\mathbb{E}_{1}^{2}$, respectively.

Corollary 5.3 ( [8], Corollary 4.9). A time-like Frenet curve $\gamma$ in $\mathbb{E}_{1}^{3}$ is a helix with $|\kappa|<|\tau|$ or $|\kappa|>|\tau|$ if and only if its space-like natural mate $\beta$ is a circle in $\mathbb{E}^{2}$ or in $\mathbb{E}_{1}^{2}$, respectively.

Corollary 5.4 ( [8], Proposition 5.3). A non-null Frenet curve $\gamma$ in $\mathbb{E}_{1}^{3}$ is a slant helix if and only if its natural mate is a general helix with $\frac{\tau_{\beta}}{\kappa_{\beta}}=\sigma_{\gamma}$, where $\sigma_{\gamma}$ is defined in (3.2).

Proposition 5.1. A non-null Frenet frame $\gamma$ with curvature $\kappa_{\gamma}$ and torsion $\tau_{\gamma}$ is a rectifying curve in $\mathbb{E}_{1}^{3}$ if and only if the curvature $\kappa_{\beta}$ and torsion $\tau_{\beta}$ of its natural mate $\beta$ satisfy

$$
\tau_{\beta}=c\left(\frac{\kappa_{\gamma}}{\kappa_{\beta}}\right)^{2}
$$

where $c$ is non-zero constant. 
Proof. Let $\gamma$ be a non-null rectifying curve with curvature $\kappa_{\gamma}$ and torsion $\tau_{\gamma}$. Then from (3.1), we can write the curve $\gamma$ as

$$
\gamma(s)=\left(s+c_{1}\right) T_{\gamma}+c_{2} B_{\gamma}
$$

for some constants $c_{1}$ and $c_{2} \neq 0$. By differentiating the last above equation, we get $c_{2} \tau_{\gamma}=$ $\left(s+c_{1}\right) \kappa_{\gamma}$ which yields

$$
\left(\frac{\tau_{\gamma}}{\kappa_{\gamma}}\right)^{\prime}=c
$$

where $c=\frac{1}{c_{2}}$. Now, we get directly (5.1) by using expressions of $\kappa_{\beta}$ and $\tau_{\beta}$. Conversely, let the condition (5.1) be hold. By substituting the expressions of $\kappa_{\beta}$ and $\tau_{\beta}$ in (5.1), we get

$$
\left(\frac{\tau_{\gamma}}{\kappa_{\gamma}}\right)^{\prime}=c
$$

for $c=\frac{1}{c_{2}}$. So we conclude $\tau_{\gamma} / \kappa_{\gamma}$ is a linear function of $s$, which say that $\gamma$ is a rectifying curve according to (3.1).

Theorem 5.1. Let $\gamma$ be a non-null Frenet curve with constant curvature $\kappa_{\gamma}=c>0$. Then its natural mate $\beta$ is a non-null spherical curve lying on sphere with radius $r=\frac{1}{c}$. The opposite is provided if $\tau_{\beta}$, the torsion of $\beta$ is a non-zero.

Proof. Since, the proof of theorem for space-like or time-like curve will be very similar to each other just for the first case will be enough to prove. Now, let $\gamma, \beta$ be two space-like Frenet curves of type1, i.e. $\varepsilon_{i}=1$ with $i=0,1,2$, and $\kappa_{\gamma}=c>0$. So from (4.1a), we have the curvature $\kappa_{\beta}$ and the torsion $\tau_{\beta}$ as

$$
\kappa_{\beta}=\sqrt{c^{2}-\tau_{\gamma}^{2}}, \quad \tau_{\beta}=\frac{c \tau_{\gamma}{ }^{\prime}}{c^{2}-\tau_{\gamma}{ }^{2}} .
$$

Now, if $\gamma$ has also a constant torsion, i.e., $\tau_{\gamma}=c_{1}$, then we directly conclude that $\kappa_{\beta}=c_{2}$ and $\tau_{\beta}=0$ of the curve $\beta$, from the above expression. Which says that $\beta$ is a part of circle with radius $c^{-1}$, so we get the natural mate $\beta$ of type1 is a spherical curve lying on a sphere of radius $c^{-1}$.

On the other hand, let the curve $\gamma$ be of a non-constant torsion. By taking into account the expressions (5.2), one can show that the following equation is provided by direct calculation

$$
\frac{\tau_{\beta}}{\kappa_{\beta}}=\left[\frac{1}{\tau_{\beta}}\left(\frac{1}{\kappa_{\beta}}\right)^{\prime}\right]^{\prime}
$$


Thus, the space-like natural mate $\beta$ of type1 is a spherical curve from (3.4). Also, we get

$$
\left(\frac{1}{\kappa_{\beta}}\right)^{2}-\left[\frac{1}{\tau_{\beta}}\left(\frac{1}{\kappa_{\beta}}\right)^{\prime}\right]^{2}=\frac{1}{c^{2}}
$$

which implies that $\beta$ lies on the sphere of radius $c^{-1}$ from (3.3).

Oppositely, assume that the natural mate $\beta$ of $\gamma$ is a spherical space-like curve, lying on the sphere of radius $c^{-1}$, with non-zero torsion, $\left(\tau_{\beta} \neq 0\right)$. So, the equation (5.4) holds. From there, we get directly

$$
\frac{\kappa_{\beta}^{\prime}}{\left|\kappa_{\beta}\right| \sqrt{c^{2}-\kappa_{\beta}^{2}}}=\frac{\tau_{\beta}}{c} .
$$

By integrating the last equation, we get

$$
\kappa_{\beta}=\frac{c}{\cosh \left(\int \tau_{\beta}(s) d s\right)} .
$$

On the other hand, from (5.2) we have

$$
\tau_{\beta}=\frac{\left(\frac{\tau_{\beta}}{\kappa_{\beta}}\right)^{\prime}}{1-\left(\frac{\tau_{\beta}}{\kappa_{\beta}}\right)^{2}} .
$$

If we substitute $\tau_{\beta}=\kappa_{\beta} \tanh \left(\theta+c_{1}\right)$ into the above expression, we obtain $\tau_{\beta}=\theta^{\prime}$. Therefore, by considering the last results in (5.5) we get

$$
\kappa_{\beta}=\frac{c}{\cosh \left(\theta+c_{1}\right)}
$$

with a suitable choice of constant.

On the other hand, by considering $\tau_{\beta}=\kappa_{\beta} \tanh \left(\theta+c_{1}\right)$ and $\kappa_{\beta}=\sqrt{\kappa_{\beta}^{2}-\tau_{\beta}^{2}}$ yields

$$
\kappa_{\beta}=\frac{\kappa_{\gamma}}{\cosh \left(\theta+c_{1}\right)}
$$

Finally, by comparing the expressions $\kappa_{\beta}$ in the equations (5.7) and (5.8) concludes the curvature $\kappa_{\gamma}=c$. So, the proof of the theorem is completed. The other cases can be proved by the same way.

Theorem 5.2. Let $\gamma$ be a non-null Frenet curve and its natural mate $\beta$ be a non-null one in $\mathbb{E}_{1}^{3}$. If the natural mate $\beta$ has constant curvature $\kappa_{\beta}=c>0$, then the relationship between the curvatures of $\gamma$ is given by

$$
\tau_{\gamma}=F(s) \kappa_{\gamma}
$$

where $F(s)$ is given by the following: 
TABLE 1. The function $F(s)$ as the Lorentzian character and type of the natural mates.

\begin{tabular}{|l|l|l|l|}
\hline$\gamma$ & $\beta$ & Necessary condition & Function $F(s)$ \\
\hline Space-like of type1 & Space-like of type1 & $\left|\kappa_{\gamma}\right|>\left|\tau_{\gamma}\right|$ & $\tanh \left(\int \tau_{\beta}(s) d s\right)$ \\
\hline Space-like of type1 & Space-like of type2 & $\left|\kappa_{\gamma}\right|<\left|\tau_{\gamma}\right|$ & $\operatorname{coth}\left(\int \tau_{\beta}(s) d s\right)$ \\
\hline Space-like of type2 & Time-like & None & $\tan \left(\int \tau_{\beta}(s) d s\right)$ \\
\hline Time-like & Space-like of type1 & $\left|\kappa_{\gamma}\right|>\left|\tau_{\gamma}\right|$ & $\operatorname{coth}\left(\int \tau_{\beta}(s) d s\right)$ \\
\hline Time-like & Space-like of type2 & $\left|\kappa_{\gamma}\right|<\left|\tau_{\gamma}\right|$ & $\tanh \left(\int \tau_{\beta}(s) d s\right)$ \\
\hline
\end{tabular}

Proof. The proof of this theorem will be proved only for the natural mates $\{\gamma, \beta\}$ being a space-like pair of type1. The other cases can be proved in the same way. Now, let the curvature of the space-like natural mate $\beta$ of $\gamma, \kappa_{\beta}$ be a constant $c>0$. By considering this assumption in the first expression in (4.1a), we get

$$
c^{2}=\kappa_{\gamma}{ }^{2}-\tau_{\gamma}{ }^{2},
$$

which yields $\kappa_{\gamma} \kappa_{\gamma}{ }^{\prime}-\tau_{\gamma} \tau_{\gamma}{ }^{\prime}=0$, where $\left|\kappa_{\gamma}\right|>\left|\tau_{\gamma}\right|$. Substituting (5.10) in the second expression in (4.1a) and then considering the last result in it, we obtain

$$
\tau_{\beta}=\frac{\tau_{\gamma}{ }^{\prime} \kappa_{\gamma}{ }^{2}-\kappa_{\gamma}{ }^{\prime} \tau_{\gamma} \kappa_{\gamma}}{\kappa_{\gamma} c^{2}}=\frac{\tau_{\gamma}{ }^{\prime}}{\kappa_{\gamma}}=\frac{\tau_{\gamma}{ }^{\prime}}{\sqrt{c^{2}+\tau_{\gamma}^{2}}} .
$$

Therefore, we get $\tau_{\gamma}=c \sinh \left(\int \tau_{\beta}(s) d s\right)$. By considering this in (5.10) yields $\kappa_{\gamma}=c \cosh \left(\int \tau_{\beta}(s) d s\right)$. Consequently, the relationship between curvatures of $\gamma$ is really as in (5.9) with the function $F(s)$ given in the first line of Table 1.

Theorem 5.3. Let $\gamma$ be a non-null Frenet curve and its natural mate $\beta$ be of constant curvature $c>0$. Then $\gamma$ is a spherical curve if and only if there is a positive constant $a \geq c$ such that the torsion $\tau_{\beta}$ of $\beta$ with respect to a suitable arc-length parametrization s satisfies one of the following:

Proof. The proof of this theorem will be proved only for the natural mates $\{\gamma, \beta\}$ being a space-like pair of type1. The other cases can be proved in the same way. Now, let the curvature of the space-like natural mate $\beta$ of $\gamma, \kappa_{\beta}$ be a constant $c>0$ and $\tau_{\beta}$ be given in the first line of Table 2, i.e,

$$
\tau_{\beta}= \pm \frac{c^{2} \sqrt{a^{2}+c^{2}} \cos (c s)}{c^{2}-\left(a^{2}+c^{2}\right) \sin ^{2}(c s)}
$$


TABLE 2. The function $\tau_{\beta}(s)$ as the Lorentzian character and type of the natural mates.

\begin{tabular}{|l|l|l|l|}
\hline$\gamma$ & $\beta$ & Necessary condition & the function $\tau_{\beta}(s)$ \\
\hline Space-like of type1 & Space-like of type1 & $\left|\kappa_{\gamma}\right|>\left|\tau_{\gamma}\right|$ & $\pm \frac{c^{2} \sqrt{a^{2}+c^{2}} \cos (c s)}{c^{2}-\left(a^{2}+c^{2}\right) \sin ^{2}(c s)}$ \\
\hline Space-like of type1 & Space-like of type2 & $\left|\kappa_{\gamma}\right|<\left|\tau_{\gamma}\right|$ & $\pm \frac{c^{2} \sqrt{a^{2}+c^{2}} \sinh (c s)}{c^{2}-\left(a^{2}+c^{2}\right) \cosh ^{2}(c s)}$ \\
\hline Space-like of type2 & Time-like & None & $\pm \frac{c^{2} \sqrt{a^{2}+c^{2}} \cosh _{(c s)}}{c^{2}-\left(a^{2}+c^{2}\right) \sinh ^{2}(c s)}$ \\
\hline Time-like & Space-like of type1 & $\left|\kappa_{\gamma}\right|>\left|\tau_{\gamma}\right|$ & $\pm \frac{c^{2} \sqrt{a^{2}+c^{2}} \sin (c s)}{c^{2}-\left(a^{2}+c^{2}\right) \cos ^{2}(c s)}$ \\
\hline Time-like & Space-like of type2 & $\left|\kappa_{\gamma}\right|<\left|\tau_{\gamma}\right|$ & $\pm \frac{c^{2} \sqrt{a^{2}-c^{2}} \cosh (c s)}{c^{2}-\left(a^{2}+c^{2}\right) \sinh (c s)^{2}}$ \\
\hline
\end{tabular}

Also, we have the following from Theorem (5.2) for the natural mates $\{\gamma, \beta\}$ being a space-like pair of type 1

$$
\tau_{\gamma}=c \sinh \left(\int \tau_{\beta}(s) d s\right), \quad \kappa_{\gamma}=c \cosh \left(\int \tau_{\beta}(s) d s\right),
$$

which gives

$$
\begin{array}{r}
\left(\frac{1}{\kappa_{\gamma}}\right)^{\prime}=-\frac{\tau_{\beta} \tanh \left(\int \tau_{\beta}(s) d s\right)}{c \cosh \left(\int \tau_{\beta}(s) d s\right)} \\
\frac{1}{\tau_{\gamma}}=\frac{1}{c \sinh \left(\int \tau_{\beta}(s) d s\right)} .
\end{array}
$$

Now, as

$$
\tanh \left( \pm \int \frac{c^{2} \sqrt{a^{2}+c^{2}} \cos (c s)}{c^{2}-\left(a^{2}+c^{2}\right) \sin ^{2}(c s)} d s\right)= \pm \frac{\sqrt{a^{2}+c^{2}}}{c} \sin (c s) .
$$

Differentiatng the above equation and using (5.12), we get

$$
\cosh ^{-2}\left(\int \tau_{\beta}(s) d s\right)=1-\left[\left(\frac{a}{c}\right)^{2}+1\right] \sin ^{2}(c s) .
$$

On the other hand, by using (5.12) and (5.16) in multiplication of (5.14a) and (5.14b), we find $\left(\frac{1}{\kappa_{\gamma}}\right)^{\prime} \frac{1}{\tau_{\gamma}}=\mp c^{-2} \sqrt{a^{2}+c^{2}} \cos (c s)$ which yields

$$
\left[\left(\frac{1}{\kappa_{\gamma}}\right)^{\prime} \frac{1}{\tau_{\gamma}}\right]^{\prime}= \pm c^{-1} \sqrt{a^{2}+c^{2}} \sin (c s) .
$$

Now, we find by considering (5.15) and (5.9) together

$$
\frac{\tau_{\gamma}}{\kappa_{\gamma}}=\tanh \left(\int \tau_{\beta}(s) d s\right)= \pm \frac{\sqrt{a^{2}+c^{2}}}{c} \sin (c s) .
$$

Finally, by considering (5.17) and (5.18) in (3.4), we conclude $\gamma$ is a space-like spherical curve of type1. 
Conversely, assume that $\gamma$ is a spherical curve and its natural mate $\beta$ has constant curvature $\kappa_{\beta}=c>0$. Then, from (3.3) and Theorem 3.4 the curvature $\kappa_{\gamma}$ and torsion $\tau_{\gamma}$ satisfy

$$
\left(\frac{1}{\kappa_{\gamma}}\right)^{2}-\left[\left(\frac{1}{\kappa_{\gamma}}\right)^{\prime} \frac{1}{\tau_{\gamma}}\right]^{2}=r^{2}
$$

where $r>0$ being constant. Thus, by substituting (5.13) and (5.14) in the last equation, we obtain

$$
\tau_{\beta} \cosh \left(\int \tau_{\beta}(s) d s\right)^{-1}= \pm \cosh \left(\int \tau_{\beta}(s) d s\right) \sqrt{a^{2}+c^{2} \cosh ^{-2}\left(\int \tau_{\beta}(s) d s\right)}
$$

where $a$ is a constant such that $a \geq c$ and $a=r c^{2}$. Thus we have

$$
\frac{c \tau_{\beta} \cosh \left(\int \tau_{\beta}(s) d s\right)^{-2}}{\sqrt{a^{2}+c^{2} \cosh \left(\int \tau_{\beta}(s) d s\right)^{-2}}}= \pm c
$$

which yields by integrating

$$
\arcsin \left(\frac{c}{a^{2}+c^{2}} \tanh \left(\int \tau_{\beta}(s) d s\right)\right)= \pm c s+c_{0}
$$

for some constant $c_{0}$. Therefore, we get

$$
\tanh \left(\int \tau_{\beta}(s) d s\right)= \pm \frac{a^{2}+c^{2}}{c} \sin (c s)
$$

after by applying a suitable translation in $s$. The last equation is equivalent to

$$
\int \tau_{\beta}(s) d s= \pm \tanh ^{-1}\left(\frac{a^{2}+c^{2}}{c} \sin (c s)\right) .
$$

Finally, by differentiating the above equation yields (5.12).

Example 5.1. Consider the unit speed space-like curve of type $2, \gamma:\left(\frac{\pi}{8}, \frac{\pi}{2}\right) \rightarrow \mathbb{E}_{1}^{3}$ given by

$$
\gamma(s)=\frac{1}{\sqrt{2}}(\ln (\sec s), s, \ln (\sec s+\tan s)) .
$$

The curvature and torsion of $\gamma$ are $\kappa_{\gamma}=\frac{\sec s}{\sqrt{2}}$ and $\tau_{\gamma}=\frac{\sec s}{\sqrt{2}}$, respectively. The Frenet frame of $\gamma$ is given by

$$
\left\{T_{\gamma}=\frac{1}{\sqrt{2}}(\tan s, 1, \sec s) ; N_{\gamma}=(\sec s, 0, \tan s) ; B_{\gamma}=\frac{1}{\sqrt{2}}(\tan s,-1, \sec s)\right\} .
$$

Thus, the time-like curve $\beta$ being natural mates of $\gamma$ is parametrized as

$$
\beta(s)=(\ln (\sec s+\tan s), c, \ln (\sec s))
$$

where $c$ is a non-zero constant. The Frenet-Serret apparatus of $\beta$ is gven by

$$
\left\{\kappa_{\beta}=\sec s ; \tau_{\beta}=0 ; T_{\beta}=(\sec s, 0, \tan s) ; N_{\gamma}=(\tan s, 0, \sec s) ; B_{\gamma}=(0,-1,0)\right\}
$$




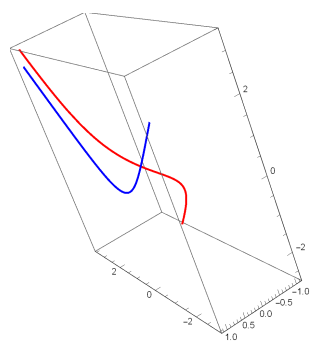

FIGURE 1. Graph of $\gamma$ (in red) and $\beta$ (in blue).

\section{REFERENCES}

[1] A.T. Ali, R. López, On slant helices in minkowski space $\mathbb{E}_{1}^{3}$, J. Korean. Math. Soc. 48 (2011), 159-167.

[2] H. Balgetir, M. Bektas, M. Ergut, Bertrand curves for nonnull curves in 3-dimensional lorentzian space, Hadronic J. 27 (2) (2004), 229-236.

[3] B.Y. Chen, When does the position vector of a space curve always lie in its rectifying plane?, Amer. Math. Monthly 110 (2) (2003), 147-152.

[4] B.Y. Chen, F. Dillen, Rectifying curves as centrodes and extremal curves, Bull. Inst. Math. Acad. Sin. 33 (2005), 77-90.

[5] J.H. Choi, T.H. Kang, Y.H. Kim, Bertrand curves in 3-dimensional space forms, Appl. Math. Comput. 219 (3) (2012), 1040-1046.

[6] J.H. Choi, T.H. Kang, Y.H. Kim, Mannheim curves in 3-dimensional space forms, Bull. Korean Math. Soc. 50 (4) (2013), 1099-1108.

[7] J.H. Choi, Y.H. Kim, Associated curves of a frenet curve and their applications, Appl. Math. Comput. 218 (18) (2012), 9116-9124.

[8] J.H. Choi, Y.H. Kim, A.T. Ali, Some associated curves of frenet non-lightlike curves in $\mathbb{E}_{1}^{3}$, J. Math. Anal. Appl. 394 (2) (2012), 712-723.

[9] S. Deshmukh, I. Al-Dayel, K. Ilarslan, Frenet curves in euclidean 4-space, Int. Electron. J Geom. 10 (2) (2017), 56-66.

[10] S. Deshmukh, B.Y. Chen, A. Alghanemi, Natural mates of frenet curves in euclidean 3-space, Turk. J. Math. 42 (5) (2018), 2826-2840.

[11] S. Deshmukh, B.Y. Chen, M.B. Turki, A differential equations for frenet curves in euclidean 3-space and its applications, Rom. J. Math. Comput. Sci. 8 (1) (2018), 1-6.

[12] K. Ilarslan, E. Nešovic, On rectifying curves as centrodes and extremal curves in the minkowski 3-space, Novi Sad J. Math. 37 (1) (2007), 53-64.

[13] K. Ilarslan, E. Nesovic, M. Petrovic-Torgasev, Some characterizations of rectifying curves in the minkowski 3-space, Novi Sad J. Math. 33 (2) (2003), 23-32.

[14] W. Kuhnel, Differential geometry: curves-surfaces-manifolds, volume 16, Weisbaden: Braunschweig, 1999.

[15] L. Kula, N. Ekmekçi, Y. Yayli, K. İlarslan, Characterizations of slant helices in euclidean 3-space, Turk. J. Math. 34 (2) (2010), 261-274. 
[16] H. Liu, F. Wang, Mannheim partner curves in 3-space, J. Geom. 88 (1-2) (2008), 120-126.

[17] B. O'neill, Semi-Riemannian geometry with applications to relativity, volume 103, Academic press, 1983.

[18] U. Pekmen, Some characterizations of lorentzian spherical space-like curves, Math. Morav. 3 (1999), $33-37$.

[19] M. Petrović-Torgašev, M. Šućurović, Some characterizations of lorentzian spherical space-like curves with the timelike and the null principal normal, Math. Morav. 4 (2000), 83-92.

[20] M. Petrović-Torgašev, M. Šućurović, Some characterizations of the lorentzian spherical timelike and null curves, Mat. Vesn. 53 (1-2) (2001), 21-27.

[21] Y.C. Wong, 'On an explicit characterization of spherical curves', Proc. Amer. Math. Soc. 34 (1) (1972), 239-242. 